\title{
AVALIAÇÃO DA RESISTÊNCIA DO CASCUDINHO (ALPHITOBIUS DIAPERINUS) (PANZER) (COLEOPTERA: TENEBRIONIDAE) A DIFERENTES TEMPERATURAS
}

\author{
F.L. Gazoni ${ }^{1}$, F. Flores ${ }^{1}$, R.A. Bampi ${ }^{1}$, F. Silveira ${ }^{1}$, R. Boufleur ${ }^{2}$, M. Lovato ${ }^{1}$
}

${ }^{1}$ Universidade Federal de Santa Maria, Departamento de Medicina Veterinária Preventiva, Laboratório Central de Diagnóstico de Patologias Aviárias, Av. Roraima, 1000, Prédio 44, Sala 5152, CEP 97105-900, Santa Maria RS, Brasil. E-mail: gazonivet@yahoo.com.br

\section{RESUMO}

\begin{abstract}
Alphitobius diaperinus, conhecido como cascudinho, é encontrado abundantemente nas instalações avícolas, e acarreta prejuízos zootécnicos e sanitários na avicultura industrial mundialmente. As formas de controle deste besouro estão baseadas exclusivamente no uso de inseticidas para os quais já existem vários relatos de populações resistentes forçando o emprego de métodos alternativos como o controle biológico, interferindo no ciclo de vida do inseto. Este estudo avaliou, em condições de laboratório, a mortalidade de larvas e adultos de $A$. diaperinus frente a diferentes temperaturas de aquecimento $\left(45^{\circ}, 50^{\circ}, 55^{\circ}\right.$ e $\left.60^{\circ} \mathrm{C}\right)$ e resfriamento $\left(-10^{\circ},-13^{\circ}\right.$ e $\left.-18^{\circ} \mathrm{C}\right)$, na presença de maravalha (PM) e ausência de maravalha (AM), através do tempo letal de $100 \%$ dos insetos $\left(\mathrm{TL}_{100}\right)$ em minutos em cada temperatura. $\mathrm{A} \mathrm{TL}_{100}$ de larvas e adultos em $45^{\circ} \mathrm{C}$ foi de 36 e $38 \mathrm{AM}$ e 42 e $57 \mathrm{PM}$, em $50^{\circ} \mathrm{C}$ foram 15 e $11 \mathrm{AM}$ e 19 e $16 \mathrm{PM}$, para $55^{\circ} \mathrm{C}$ foi de 8 e $9 \mathrm{AM}$ e $12 \mathrm{PM}$ e em $60^{\circ}$ $\mathrm{C}$ foi de 5 e 6 AM e 11 e 9 PM. Já em $-10^{\circ} \mathrm{C}$ variou de 57 e 60 AM e 70 e 75 PM, em - $13^{\circ}$ C levou 52 e $55 \mathrm{AM}$ e 60 e $64 \mathrm{PM}$ e em $-18^{\circ} \mathrm{C}$ levou 42 e 43 AM e 51 e 52 PM. Em todas as temperaturas avaliadas houve mortalidade dos insetos em tempo consideravelmente rápido e métodos de controle, através da temperatura, devem levar em consideração esses pontos para referência.
\end{abstract}

PALAVRAS-CHAVE: Controle, variação ambiental, mortalidade, fermentação.

\begin{abstract}
EVALUATION OF THE RESISTANCE OF MEALWORMS (ALPHITOBIUS DIAPERINUS) (PANZER) (COLEOPTERA: TENEBRIONIDAE) AT DIFFERENT TEMPERATURES. Alphitobius diaperinus, known as the lesser mealworm, is abundantly found in poultry houses, and causes losses in poultry worldwide. The ways to control this beetle are based exclusively on the use of insecticides, concerning which there are already several reports of resistant populations, thus forcing the use of alternative methods such as biological control, interfering in the life cycle of the insect. The present study evaluated, under laboratory conditions, the mortality of larvae and adults of $A$. diaperinus at different temperatures of heating $\left(45^{\circ}, 50^{\circ}, 55^{\circ}\right.$ and $\left.60^{\circ} \mathrm{C}\right)$ and cooling $\left(-10^{\circ},-13^{\circ}\right.$ and $\left.-18^{\circ} \mathrm{C}\right)$ in the presence (PM) and absence of wood shavings (AM) by way of the lethal time of $100 \%$ of insects $\left(\mathrm{TL}_{100}\right)$ in minutes at each temperature. The $\mathrm{TL}_{100}$ for larvae and adults at $45^{\circ} \mathrm{C}$ were 36 and $38 \mathrm{AM}$ and 42 and $57 \mathrm{PM}$, at $50^{\circ} \mathrm{C}$ they were 15 and $11 \mathrm{AM}$ and 19 and $16 \mathrm{PM}$, at $55^{\circ} \mathrm{C}$ they were 8 and $9 \mathrm{AM}$ and $12 \mathrm{PM}$, and at $60^{\circ} \mathrm{C}$ they were 5 and $6 \mathrm{AM}$ and 11 and $9 \mathrm{PM}$. At $-10^{\circ} \mathrm{C}$ they were 57 and $60 \mathrm{AM}$ and 70 and $75 \mathrm{PM}$, at $-13^{\circ} \mathrm{C}$ they were 52 and $55 \mathrm{AM}$ and 60 and $64 \mathrm{PM}$, and at $-18^{\circ} \mathrm{C}$ they were 42 and $43 \mathrm{AM}$ and 51 and $52 \mathrm{PM}$. At all temperatures, the mortality of the insects was quick; therefore methods of control by way of temperature should consider these points for reference.
\end{abstract}

KEY WORDS: Control, environmental variation, mortality, fermentation.

\section{INTRODUÇÃO}

Alphitobius diaperinus é, atualmente, um dos grandes problemas da avicultura mundial sendo também conhecido como praga secundária de derivados de grãos armazenados. Adaptou-se muito bem às condições dos aviários, onde se alimenta de ração, fezes e de animais mortos (McAllister et al.,1995; PACHECO; PAULA, 1995).

Este inseto desempenha um papel importante na transmissão denumerosos agentes patogênicos como vírus(EIDSONetal., 1966;SNEDEKER et al., 1967; GOODWIN; WALTMAN, 1996; WATSON et al., 2000), fungos (De LAS CASAs et al., 1972), protozoários (GoODWIN; WATMAN,

${ }^{2}$ Universidade Federal do Rio Grande do Sul, Departamento de Medicina Animal, Porto Alegre, RS, Brasil. 
1996; Huber et al., 2007) e helmintos (KARUNAMOORTHY et al., 1994). Além disso, o besouro pode servir como fonte de infecção para Campylobacter spp. (BATES et al., 2004), Escherichia coli, Salmonella spp. e várias outras bactérias (McAlLISTER et al., 1994; CHERNAKI-LEFFER et al., 2002; Segabinazi et al., 2005; VitTori et al., 2007; ChernaKi-LeFFer, 2011).

O controle desta praga está vinculado ao uso de inseticidas, piretroides e organofosforados, e é crescente o número de relatos de populações resistentes a estes compostos (LAMBKIN, 2005; LAMBKIN; RICE, 2006; HAMm et al., 2006; KAUfMAN et al., 2008; TOMBERLIN et al., 2008), inclusive no Brasil (CHERNAKI-LEFFER, 2011).

Em vista disso, a busca por métodos de controle alternativos aos métodos convencionais apresenta-se necessária e em especial aqueles métodos que não gerem danos à sanidade das aves e à saúde humana; levando em consideração também o ciclo biológico do inseto.

A maioria dos estudos que relacionam a temperatura e os estágios de desenvolvimento do $A$. diaperinus leva em consideração o intervalo entre $20^{\circ} \mathrm{C}$ e $38^{\circ}$ C (Rueda; Axtell, 1996). Poucos são os estudos que avaliam o comportamento desse inseto frente a temperaturas extremas e os que existem avaliaram principalmente baixas temperaturas, pois foram realizados na Europa, onde facilmente se atinge temperaturas negativas por longos períodos no inverno, visando um controle natural dessa praga (SALIN et al., 1998; RenAult et al., 1999; RenAult et al., 2006; KošTÁL et al., 2007). SALIN et al. (1998) demonstraram que adultos de $A$. diaperinus podem ser controlados a partir de tratamentos térmicos que ultrapassem $48^{\circ}$ C, no entanto, estes autores não avaliaram estágios importantes como a fase larval do inseto.

É comum, nas regiões Norte e Nordeste do Rio Grande do Sul, se atingir temperaturas abaixo de $0^{\circ} \mathrm{C}$ durante longo período, e são nessas mesmas regiões onde se localizam os principais polos de avicultura do estado.

Contudo, este estudo teve como objetivo avaliar a resistência da fase larval e adulta do A. diaperinus, provenientes de aviários da região Norte do Rio Grande do Sul, frente a diferentes temperaturas de aquecimento e resfriamento.

\section{MATERIAL E MÉTODOS}

Os insetos foram coletados juntamente com a cama de maravalha, em aviários comerciais da região Norte do Estado do Rio Grande do Sul e enviados ao Laboratório Central de Diagnóstico de Patologias Aviárias(LCDPA), ondeforammantidos em recipientes plásticos, com tampa perfurada, alimentados com ração do aviário onde foram realizadas as coletas e mantidos em temperatura ambiente durante sete dias.
Para a realização dos experimentos, de forma aleatória, os insetos foram separados e organizados em dois grupos: larvas e adultos, com 20 insetos cada e subdivididos em placas com presença (PM) e ausência de maravalha (AM), sempre em triplicata. Estes foram aclimatados a $22^{\circ} \mathrm{C}$ por $36 \mathrm{~h}$ antes da exposição às baixas e altas temperaturas.

Os insetos foram submetidos às temperaturas de: $-18^{\circ} \mathrm{C},-13^{\circ} \mathrm{C}-10^{\circ} \mathrm{C}, 45^{\circ} \mathrm{C}, 50^{\circ} \mathrm{C}, 55^{\circ} \mathrm{C}$ e $60^{\circ} \mathrm{C}$, em câmaras climatizadas, sendo monitorado visualmente o tempo para obtenção da mortalidade total dos insetos $\left(\mathrm{TL}_{100}\right)$.

Após isso, as placas foram retiradas dos respectivos locais de incubação e deixadas em temperatura ambiente por 20 minutos. Durante esse intervalo de tempo eram agitadas com movimentos circulares, a cada cinco minutos, para avaliar a movimentação, o que indicava que o inseto (adulto ou larva) estava vivo. Após um dia, se os adultos continuavam em decúbito dorsal e sem movimentação das patas e as larvas com paralisa do movimento corporal, eles eram considerados mortos.

O teste AM foi realizado para simular um ambiente onde não há proteção, semelhante o encontrado pelos cascudinhos no ambiente externo ao sistema de criação das aves. Já o teste PM simulou um ambiente que poderia ser encontrado nas granjas de produção, no qual a cama das aves torna-se um isolante que reproduz um microclima ideal para o seu desenvolvimento.

A análise estatística foi realizada pelo teste de Qui-quadrado $\left(X^{2}\right)$ utilizando o programa SAS 9.2 de 2010 com intervalo de confiança de 95\%.

\section{RESULTADOS E DISCUSSÃO}

Conforme demonstrado na Tabela 1, o tempo letal de $100 \%$ dos insetos $\left(\mathrm{TL}_{100}\right)$ em $-10^{\circ} \mathrm{C}$ variou de 57 a 60 minutos, sem a utilização de maravalha (AM) nas placas e 70 a 75 minutos com a utilização de maravalha (PM) nas placas para larvas e adultos, respectivamente. $\mathrm{O} \mathrm{TL}_{100} \mathrm{em}-13^{\circ} \mathrm{C}$ foi de $52 \mathrm{e}$ 55 minutos AM e 60 e 64 minutos PM para larvas e adultos, respectivamente. Já o $\mathrm{TL}_{100}$ para $-18^{\circ} \mathrm{C}$ foi de 42 e 43 minutos AM e 51 e 52 minutos PM para larvas e adultos, respectivamente.

Na análise estatística $\left(X^{2}\right)$ não foram encontradas diferenças significativas utilizando a de $5 \%$ para as médias dos resultados de larvas e adultos na AM e PM e também na avaliação de larvas versus adultos, AMePMversus o tempo de exposição, ou seja, todas essas temperaturas podem matar ou interromper $\mathrm{o}$ ciclo do A. diaperinus em um curto intervalo de tempo.

Observou-se que, nessas temperaturas, os insetos paralisaram sua movimentação, primeiramente em AM, comparado aos insetos que estavam em PM, sen- 
doa maravalha desempenhandoum fator de proteção para os insetos. O tempo de mortalidade nas placas com maravalha foi maior em todas as temperaturas com relação a não utilização desse substrato. Logo após a retirada das placas do resfriamento, as larvas começaram a se movimentar antes dos adultos e a mortalidade ocorreu num intervalo de tempo menor.

RENAULT et al. (1999) observaram que o tempo letal $50\left(\mathrm{TL}_{50}\right)$ variou de 9,5 dias a $6^{\circ} \mathrm{C}$ para 47,6 dias a $10^{\circ} \mathrm{C}$ e tempo letal $99\left(\mathrm{TL}_{99}\right)$ variou de 20 dias a $6^{\circ} \mathrm{C}$ para 102,2 dias a $10^{\circ} \mathrm{C}$. A sobrevida média não mostra diferenças significativas entre os sexos, mas a $10^{\circ} \mathrm{C}$ todos os machos são mortos após 88 dias e todas as fêmeas, após 107 dias. Em virtude disto, esse experimento não realizou a sexagem dos insetos, pois os autores mencionam em seu trabalho que não haveria diferenças significativas entre sexos em temperaturas abaixo de $6^{\circ} \mathrm{C}$.

A temperatura de $6^{\circ} \mathrm{C}$ é progressivamente letal para os adultos de A. diaperinus; a atividade cessa e todos os insetos entram em estado chamado de coma pelo frio ou, em inglês, Chill-Coma (CC). OCCéum estado reversível, mas os insetos que entram nesta fase não se recuperam na maioria dos casos (RENAULT etal.,1999). No Rio Grande doSul, durante o inverno, quando ocorre o período de vazio sanitário, o A. diaperinus é submetido a temperaturas abaixo de $0^{\circ} \mathrm{C}$, assim como a sensação térmica pode chegar a $-10^{\circ} \mathrm{C}$ principalmente durante a madrugada. Esse período sanitário pode variar de dois a sete dias dependendo da estratégia utilizada pela empresa avícola com seus integrados. Desta forma, se os intervalos fossem concomitantes com os períodos mais frios, a taxa de sobrevivência de insetos seria menor para o próximo lote, especialmente na primeira semana de vida dos pintainhos, pois é a fase que se mostra mais importante para o desempenho zootécnico das aves e mais suscetível aos problemas sanitários envolvendo o $A$. diaperinus.

Em relação às temperaturas de aquecimento, a $\mathrm{TL}_{100}$ a $45^{\circ} \mathrm{C}$ de larvas e adultos foi de 36 e $38 \mathrm{~min}$ AM e 42 e 57 min PM. Em $50^{\circ} \mathrm{C}$ foram necessários 15 e 11 min $A M$ e 19 e 16 min $\mathrm{PM}_{\text {para }} \mathrm{TL}_{100}$ de larvas e adultos, respectivamente. $\mathrm{OTL}_{100}$ para $55^{\circ} \mathrm{C}$ foi de 8 e 9 min AM e 12 min PM para larvas e adultos. Já o $\mathrm{TL}_{100}$ para $60^{\circ} \mathrm{C}$ foi de 5 e 6 min sem a utilização de maravalha nas placas e 11 e 9 min com a utilização de maravalha nas placas para larvas e adultos, respectivamente, conforme demonstrado na Tabela 2.

Nestas temperaturas ocorreu movimentação dos insetos nas placas e essa agitação foi acelerada com o aumento da temperatura, sendo esse comportamento relacionado à tentativa de fuga. Os insetos adultos que se encontravam em um estágio debilitado paralisaram os movimentos, exteriorizaram suas asas e se esconderam entre a maravalha. As larvas apresentavam movimentos desordenados, desorientados e em espiral, as quais apresentaram maior resistência se comparado aos adultos em alguns intervalos de tempo.

Tabela 1 - Percentuais de larvas e adultos mortos no resfriamento, nos diferentes intervalos de tempo, em placas de Petri, sem e com maravalha.

\begin{tabular}{|c|c|c|c|c|c|c|}
\hline \multirow{3}{*}{ Temperatura $\left({ }^{\circ} \mathrm{C}\right)$} & & & \multicolumn{4}{|c|}{ Temperatura $\left({ }^{\circ} \mathrm{C}\right)$} \\
\hline & \multicolumn{2}{|c|}{ Tempo (minutos) } & \multicolumn{2}{|c|}{ Sem maravalha } & \multicolumn{2}{|c|}{ Com maravalha } \\
\hline & Larvas & Adultos & Larvas & Adultos & Larvas & Adultos \\
\hline-10 & 57 & 60 & 100 & 100 & 65 & 75 \\
\hline-10 & 70 & 75 & 100 & 100 & 100 & 100 \\
\hline-13 & 52 & 55 & 100 & 100 & 75 & 40 \\
\hline-13 & 60 & 64 & 100 & 100 & 100 & 100 \\
\hline-18 & 42 & 43 & 100 & 100 & 80 & 70 \\
\hline-18 & 51 & 52 & 100 & 100 & 100 & 100 \\
\hline
\end{tabular}

Tabela 2 - Percentuais de larvas e adultos mortos em aquecimento, nos diferentes intervalos de tempo, em placas de Petri, sem e com maravalha.

\begin{tabular}{|c|c|c|c|c|c|c|}
\hline \multirow{3}{*}{ Temperatura $\left({ }^{\circ} \mathrm{C}\right)$} & & & \multicolumn{4}{|c|}{ Mortalidade (\%) } \\
\hline & \multicolumn{2}{|c|}{ Tempo (minutos) } & \multicolumn{2}{|c|}{ Sem maravalha } & \multicolumn{2}{|c|}{ Com maravalha } \\
\hline & Larvas & Adultos & Larvas & Adultos & Larvas & Adultos \\
\hline 45 & 36 & 38 & 100 & 100 & 0 & 0 \\
\hline 45 & 42 & 57 & 100 & 100 & 100 & 100 \\
\hline 50 & 15 & 11 & 100 & 100 & 0 & 0 \\
\hline 50 & 19 & 16 & 100 & 100 & 100 & 100 \\
\hline 55 & 8 & 9 & 100 & 100 & 0 & 0 \\
\hline 55 & 12 & 12 & 100 & 100 & 100 & 100 \\
\hline 60 & 5 & 6 & 100 & 100 & 0 & 0 \\
\hline 60 & 11 & 9 & 100 & 100 & 100 & 100 \\
\hline
\end{tabular}


Salinetal. (1998) demonstraram que A. diaperinus adultos, em torno de $0 \%$ de umidade relativa entre 20 e $40^{\circ} \mathrm{C}$, tem aumentada a taxa de perda de água corporal gradualmente e acima de $40^{\circ} \mathrm{C}$ o fluxo de transpiração aumenta abruptamente coincidindo com o início de uma vigorosa atividade locomotora. Este ponto crítico corresponde à abertura dos espiráculos, a partir do qual a água é expelida do sistema traqueal. Através disto, acredita-se que este seja o motivo pelo qual houve a observação, neste experimento, de paralisia dos adultos e, possivelmente, explica a mortalidade ocasionada pela desidratação dos insetos.

Conforme RuEDA; Axtell (1996), a temperatura ideal de desenvolvimento é de $35^{\circ} \mathrm{C}$ e para chegar à maior mortalidade seria necessário um maior período de exposição. Neste sentido, um estudo demonstra que é possível controlar a população de adultos de A. diaperinus quando é atingida a temperatura de $48^{\circ} \mathrm{C}$ (SAlin et al. 1998), porém, o estágio larval não havia sido avaliado nestas condições.

Contudo, através deste experimento, foi possível notar que a partir de $45^{\circ} \mathrm{C}$ existe mortalidade tanto de larvas como de adultos, corroborando com estudos já realizados e abrindo perspectivas para que o controle térmico seja também empregado em um programa de controle estratégico do inseto.

É importante lembrar que, atualmente, o método de controle empregado frente esta praga é baseado em inseticidas, principalmente da família dos piretroides e organofosforados, para os quais existem vários relatos de populações resistentes, inclusive no Brasil (CHERNAKI-LeFFER, 2011). Outras propostas como o emprego de gás fosfina (GAZONi et al., 2011) e a utilização de fungos entomopatogênicos (SANTORO et al., 2010) vêm sendo propostas como método de controle; porém, apesar de demonstrarem resultados animadores, ainda estão longe de serem aplicados devido a problemas de difícil manejo, como no caso da utilização de gás fosfina, e de eficiência dos fungos num ambiente tão adverso ao seu desenvolvimento como é o galpão avícola, pela alta concentração de amônia, temperatura elevada eumidadeinadequada.

Como uma alternativa viável, as fermentações de cama se mostram interessantes formas de manejo e reutilização do substrato aviário e atingem, na maioria das vezes, uma temperatura de $60^{\circ} \mathrm{C}$ (FIORENTIN, 2005). JEFFREY et al. (1998) demonstraram que o empilhamento da cama pode atingir $50^{\circ} \mathrm{C}$, porém, não uniformemente. Entretanto o método de fermentação com lona por toda a extensão do galpão pode atingir facilmente essas temperaturas.

Neste sentido, SILVA et al. (2007) avaliaram diferentes métodos de tratamento da cama aviária para sua reutilização e demonstraram que a melhor opção foi a vedação com lona por toda a extensão do aviário. Além disso, os mesmos autores observaram que houve uma diminuição significativa no número de enterobactérias (UFC/g) e bactérias mesófilas na cama e, apesar de não haverem feito uma avaliação da mortalidade decascudinhos através deste método, observaram enorme presença de insetos mortos no final do experimento, chamando atenção para uma avaliação da fermentação como forma de controlar populações de $A$. diaperinus no galpão.

\section{CONCLUSÃO}

Nesse experimento, temperaturas abaixo de $-10^{\circ}$ C e acima de $45^{\circ} \mathrm{C}$ causam a mortalidade tanto de larvas como adultos de $A$. diaperinus na presença e ausência de maravalha.

Mais estudos são necessários para avaliar formas de controle biológico de populações de $A$. diaperinus, utilizando métodos de regulação térmica.

\section{AGRADECIMENTOS}

Os autores gostariam de agradecer ao professor Paulo Pacheco, do Departamento de Zootecnia, UFSM, pelo o auxílio na análise estatística deste experimento.

\section{REFERÊNCIAS}

BATES, C.; HIETT, K.L.; STERN, N.J. Relationship of Campylobacter isolated from poultry and from darkling beetles in New Zealand. Avian Diseases, v.48, p.138-147, 2004.

CHERNAKI-LEFFER, A.M.; BIESDORF, S.M.; ALMEIDA, L.M.; LEFFER, E.V.B.; VIGNE, F. Isolamento de enterobactérias em Alphitobius diaperinus e na cama de aviários no Oeste do Estado do Paraná, Brasil. Revista Brasileira de Ciência Avícola, v.4, n.3, p.243-247, 2002.

CHERNAKI-LEFFER, A.M.; SOSA-GOMÉZ, D.R.; ALMEIDA, L.M.; LOPES, I.O.N. Susceptibility of Alphitobius diaperinus (Panzer) (Coleoptera, Tenebrionidae) to cypermethrin, dichlorvos and triflumuron in southern Brazil. Revista Brasileria de Entomologia, v.55, p.125-128, 2011.

DE LAS CASAS, E.; HAREIN, P.K.; POMEROY, B.S. Bacteria and fungi within the lesser mealworm collected from poultry brooder houses. Environmental Entomology, v.1, p.27-30, 1972.

EIDSON, C.S.; SCHMITTLE, S.C.; GOODE, R.B.; LAL, J.B. Induction of leucosis tumors with the beetle Alphitobius diaperinus. American Journal of Veterinary Research, v.27, n.119, p.1053-1057, 1966. 
FIORENTIN. L. Aspectos bacteriológicos da reutilização da cama de aviários de frangos de corte. Revista Nordeste Rural. Disponível em: <http:/ / www.nordesterural.com.br/ redir.asp?instanceld $=106 \&$ moduleObjld $=2833 \& u r l=$ / nordesterural/matler.asp \%3Fnewsld \%3D2833> acesso em: 6 de set. 2005 .

GAZONI, F.; WILSMANN, C.G.; FLORES, F.; SILVEIRA, F.; BAMPI, R.A.; BOUFLER, R. LOVATO, M. Efficacy of phosphine gas against the darkling beetle (Alphitobius diaperinus). Acta Scientiae Veterinariae, v.39, art. 965, 2011.

GOODWIN, M.A.; WALTMAN, W.D. Transmission of Eimeria, viruses, and bacteria to chicks: darkling beetles (Alphitobius diaperinus) as vectors of pathogens. Journal of Applied Poultry Cattle, v.5, p.51-55, 1996.

HAMM, R.L.; KAUFMAN, P.E.; REASOR, C.A.; RUTZ, D.A.; SCOTT, J.G. Resistance to cyfluthrin and tetrachlorvinphos in the lesser mealworm, Alphitobius diaperinus, collected from the eastern United States. Pest Management Science, v.62, p.673-677, 2006.

HUBER, K.; GOUILLOUD, L.; ZENNER, L. A preliminary study of natural and experimental infection of the lesser mealworm Alphitobius diaperinus (Coleoptera: Tenebrionidae) with Histomonas meleagridis (Protozoa: Sarcomastigophora). Avian Pathology, v.36, p.279-282. 2007.

JEFFREY, J.S.; KIRK, J.H.; ATWILL, E.R.Y.; CULLOR, J.S. Research notes: Prevalence of selected microbial pathogens in processed poultry waste used as dairy cattle feed. Poultry Science, v.77, p.808-811, 1998.

KARUNAMOORTHY, G.; CHELLAPPA, D.J.; ANANDAN, R. The life history of Subulura brumpti in the beetle Alphitobius diaperinus. Indian Veterinary Journal, v.71, p.12-15, 1994.

KAUFMAN, P.E.; STRONG, C.; RUTZ, D.A. Susceptibility of lesser mealworm (Coleoptera: Tenebrionidae) adults and larvae exposed to two commercial insecticides on unpainted plywood panels. Pest Management Science, v.64, p.108-111, 2008.

KOŠTÁL, V.; Renault, D.; Mehrabianová, A.; Bastl, J. Insect cold tolerance and repair of chill-injury at fluctuating thermal regimes: Role of ion homeostasis. Comparative Biochemistry and Physiology - A Molecular and Integrative Phisiology, v.147, n.1, p.231-238, 2007.

LAMBKIN, T.A. Baseline responses of adult Alphitobius diaperinus (Panzer) (Coleoptera: Tenebrionidae) to fenitrothion, and susceptibility status of populations in Queensland and New South Wales, Australia. Journal of Economic Entomology, v.98, p.938-942, 2005.

LAMBKIN, T.A.; RICE, S.J. Baseline responses of Alphitobius diaperinus (Coleoptera: Tenebrionidae) to cyfluthrin and detection of strong resistance in field populations in eastern Australia. Journal of Economic Entomology, v.9, p.908-913, 2006.

McALLISTER, J.C.; STEELMAN, C.D.; SKEELES, J.K. Reservoir competence of the lesser mealworm (Coleoptera: Tenebrionidae) for Salmonella Typhimurium (Eubacteriales:Enterobacteriaceae). Journal of Medical Entomology, v.31, p.369-372, 1994.

MCALLISTER, J.C.; STEELMAN, C.D.; NEWBERRY, L.A.; SKEELES, J.K. Isolation of infectious bursal disease virus from the lesser mealworm, Alphitobius diaperinus (Panzer). Poultry Science, v.74, n.1, p.45-49, 1995.

PACHECO, I.A.; PAULA, D.C. Insetos de grãos armazenados - Identificação e biologia. Campinas: Fundação Cargil, 1995. 11p.

RENAULT, D.; SALINA, C.; VANNIERB, G.; VERNONA, P.; Survival and chill-coma in the adult lesser mealworm, Alphitobius diaperinus (Coleoptera: Tenebrionidae), exposed to low temperatures. Journal of Thermal Biology, v.24, p.229-236, 1999.

RENAULT, D.; BOUCHEREAU, A.; DELETTRE, Y.R.; HERVANT, F.; VERNON, P. Changes in free amino acids in Alphitobius diaperinus (Coleoptera: Tenebrionidae) during thermal and food stress. Comparative Biochemistry and Physiology - A Molecular and Integrative Phisiology, v.143, p279-285, 2006.

RUEDA, L.M.; AXTELL, R.C. Temperature-dependent Development and Survival of the Lesser Mealworm, Alphitobios diaperinus. Poultry Science, v.10, p.80-86, 1996.

SALIN, C.; VERNON, P.; VANNIER, G. The supercooling and high temperature stupor points of the adult lesser mealworm Alphitobius diaperinus (Coleoptera: Tenebrionidae). Journal of Stored Products Research, v.34, p.385-394, 1998.

SANTORO, P.H.; NEVES, P.M.O.J.; AMARO, J.T.; CONSTANSKI, K.; LÓPEZ, E.A.L.; ALVES, L.F.A. Association of inert powders with the entomopathogenic fungi for darkling beetle (Alphitobius diaperinu) control. Ciência Rural, v.40, n.6, p.1354-1359, 2010.

SAS INSTITUTE. Version 9.2. Cary, NC.: SAS, 2010.

SEGABINAZI, S.D.S.; FLORES, M.L.; SILVA BARRETOS, A.; JACOBSEN, G.; ELTZ, R.D. Bactérias da família enterobacteriaceae em Alphitobius diaperinus oriundos de granja avícolas dos estados do Rio Grande do Sul e Santa Catarina. Acta Scientiae Veterinariae, v.33, n.1, p.51-55, 2005.

SILVA, V.S.; VOSS, D.; COLDEBELLA, A.; BOSSETTI, N.; AVILLA, V.S. Efeito de tratamentos sobre a carga bacteriana de cama de aviário reutilizada em frangos de corte. Concórdia: Embrapa, 2007. 10p. (Documento Tecnico 467). Disponível em: <http:/ / www.cnpsa.embrapa.br/sgc/sgc_publicacoes/publicacao_k1b2010q.pdf> Acesso em: 14 nov. 2011. 
SNEDEKER, C.; WILLS, F.K.; MOULTHROP, I.M. Some studies on the infectious bursal agent. Avian Disease, v.11, p.519-528, 1967.

TOMBERLIN, J.K.; RICHMAN, D.; MYERS, H.M. Susceptibility of Alphitobius diaperinus (Coleoptera: Tenebrionidae) from Broiler Facilities in Texas to Four Insecticides. Journal of Economic Entomology, v.101 n.2, p.480-483, 2008.

VITTORI, J.; SCHOCKEN-ITURRINO, P.; TROVÓ, K.P.; RIBEIRO, C.A.M.; BARBOSA, G.G.; SOUZA, L.M.; PIGATTO, C.P. Alphitobius diaperinus como veiculador de Clostridium perfrigens em granjas avícolas do interior paulista - Brasil. Ciência Rural, v.37, n.3, p.894-896, 2007.

WATSON, D.W.; GUY, J.S.; STRINCHAM, S.M.

Limited Transmission of Turkey Coronavirus in Yong Turkeys by Adult Alphitobius diaperinus (Coleoptera:Tenebrionidae). Journal of Medical Entomology, v.37, p.480-483, 2000.

Recebido em 26/7/10

Aceito em 30/11/11 